\title{
感 Jefferson. \\ Does 17 Alpha-Hydroxyprogesterone Caproate Decrease the Rate of Preterm Birth in Women with an Ultrasound-Indicated Cerclage?
}

Rafael TJ1, Mackeen D1, Berghella V1

${ }^{1}$ Division of Maternal-Fetal Medicine, Department of Obstetrics and Gynecology, Jefferson Medical College, Thomas Jefferson University, Philadelphia, PA

\section{ABSTRACT}

Objective To estimate whether the use of 17 alpha-hydroxyprogesterone caproate (17P) in women (P) reduces the rate of preterm birth (PTB).

Methods Retrospective cohort study of women with a 1) previous spontaneous PTB between 20-36 study group consisted of women treated with $17 \mathrm{P}$ starting at 16-20 weeks, and the control group consisted of women who were not treated with 17P. Primary outcome was PTB $<35$ weeks. Secondary Results 59 women with a prior PTB and current UIC were identified. 15 women (25.4\%) received 17P, (1) shortest $C L$ prior to cerclage) did not differ between the two groups. 17P was not associated with significant effects on the primary or secondary outcomes.

Conclusion Among women with a prior spontaneous PTB and current UIC for CL $<25 \mathrm{~mm}$, 17P did not educe the rate of PTB $<35$ weeks.

\section{BACKGROUND}

- Placing an ultrasound-indicated cerclage (UIC) once the transvaginal cervical length (TV CL) falls below $25 \mathrm{~mm}$ between 16-23 6/7 weeks' gestation in women with a previous preterm birth (PTB) has been shown to reduce the rate of PTB.

- In women with a prior PTB, weekly 17 alpha-hydroxyprogesterone caproate (17P) has also been shown to be beneficial in reducing the rate of PTB.

- While the most recent randomized study on UIC involved many women receiving 17P, the earlier studies did not have women on 17P, as at that time, it was not yet standard of care.

- Our objective was to examine the efficacy of $17 \mathrm{P}$ at prolonging gestation in women with a prior PTB, who have received an UIC.

\section{MATERIALS \& METHODS}

- This was a retrospective cohort study, using data from an existing prematurity database (1995-2009).

- Inclusion criteria: singleton gestation, previous spontaneous PTB between 20-36 6/7 weeks, asymptomatic women with TV CL $<25 \mathrm{~mm}$, with or withou usage of 17P, UIC placed between 15-23 6/7 weeks.

- Exclusion criteria: multiple gestations, history or physical-exam indicated cerclage, indicated PTB, major fetal anomalies.

- The primary outcome variable was PTB $<35$ weeks. Secondary outcomes included PTB $<32$ weeks, PTB $<37$ weeks, GA at delivery, and neonatal birthweight.

\section{RESULTS}

59 women with a prior PTB and current UIC were identified:

$$
\text { 17P: } 15(25.4 \%) \text { No 17P: } 44(74.6 \%)
$$

- Baseline characteristics (Table 1) did not differ between the two groups.

Table 1: Demographics

\begin{tabular}{|c|c|c|c|c|}
\hline \multicolumn{2}{|r|}{ Variable } & $\begin{array}{c}\text { 17P } \\
n=15(\%)\end{array}$ & $\begin{array}{l}\text { No 17P } \\
n=44(\%)\end{array}$ & $\mathbf{p}$ \\
\hline \multicolumn{2}{|l|}{ Age $^{*}$} & 28 [25-33] & 28 [25-33] & 0.85 \\
\hline \multirow{2}{*}{ Race } & African American & $14(93.3)$ & $32(72.7)$ & \\
\hline & Caucasian & $1(6.7)$ & $12(27.3)$ & 0.15 \\
\hline \multicolumn{2}{|c|}{ Smoking } & $3(20.0)$ & $11(25.0)$ & 0.99 \\
\hline \multicolumn{2}{|c|}{$>1$ Dilation and Curettage } & $3(20.0)$ & $11(25.0)$ & 0.99 \\
\hline \multicolumn{2}{|c|}{ History of Cone Biopsy } & $3(20.0)$ & $2(4.5)$ & 0.10 \\
\hline \multicolumn{2}{|c|}{ History of $>1$ PTB } & $6(40.0)$ & $16(36.4)$ & 0.80 \\
\hline \multicolumn{2}{|c|}{ Earliest GA (wk) of Prior PTB* } & $25[20-32]$ & $22[20-24]$ & 0.06 \\
\hline \multicolumn{2}{|c|}{ GA (wk) at Cerclage Placement* } & 21 [16-22] & 19 [17-21] & 0.38 \\
\hline \multicolumn{2}{|c|}{ Pre-Cerclage Shortest CL $(\mathrm{mm})^{*}$} & $18[12-22]$ & $17[12-20]$ & 0.64 \\
\hline
\end{tabular}

- 17P was not associated with significant effects on the primary or secondary outcomes (Table 2, Figures 1 and 2).

Table 2: Delivery Outcomes*

Median gestational age at delivery ( $p$ value 0.17$)$

17P 35 weeks (27-38 wks)

No 17P 38 weeks (33-39 wks)

Median neonatal birthweight ( $p$ value 0.50 )

17P 2381g (1724-3196g)

No 17P 2920g (1800-3380g) email: timothyrafael@hotmail.com Figure 1: PTB outcomes (17P vs No 17P)

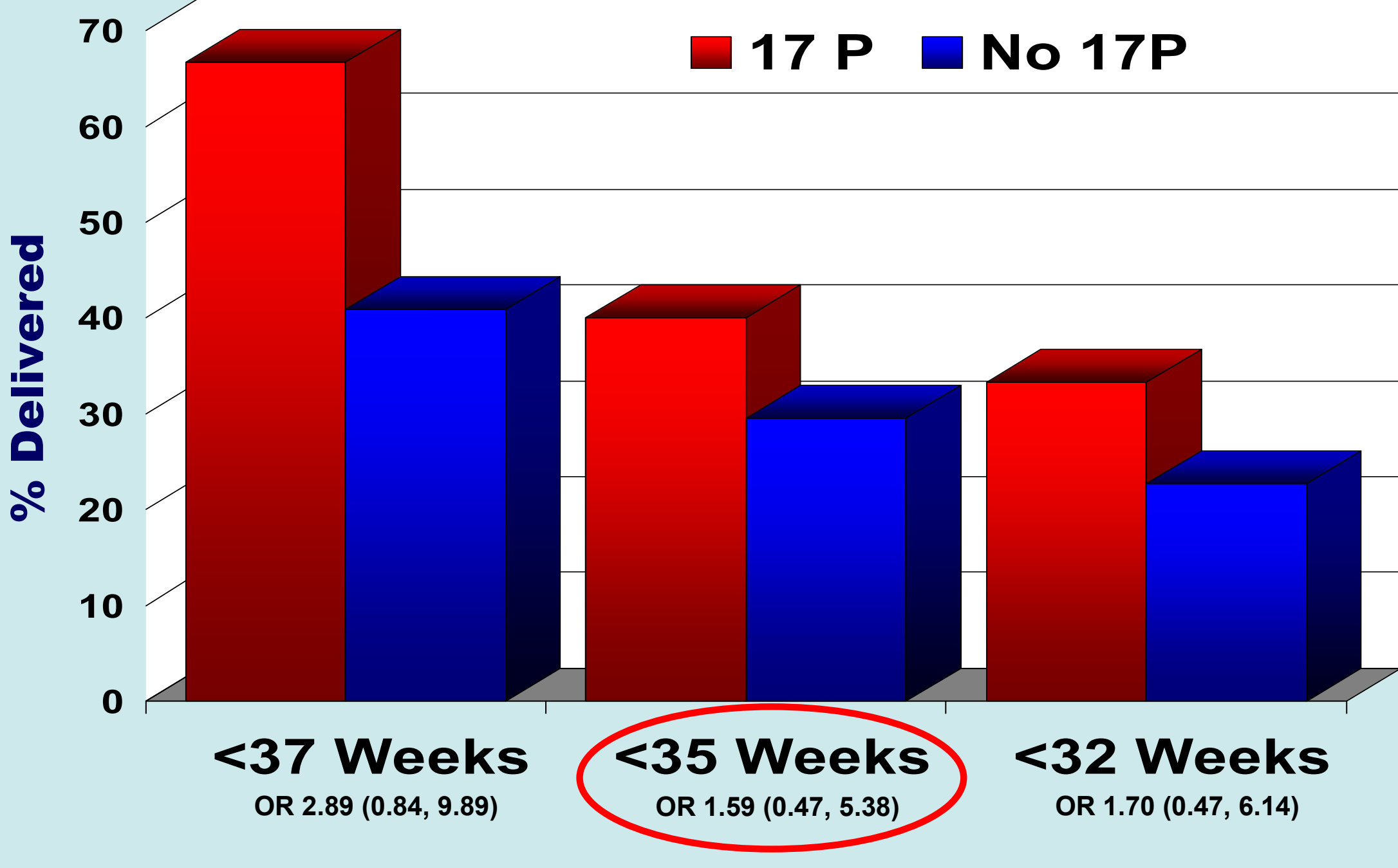

Figure 2: Kaplan-Meier (17P vs No 17P)

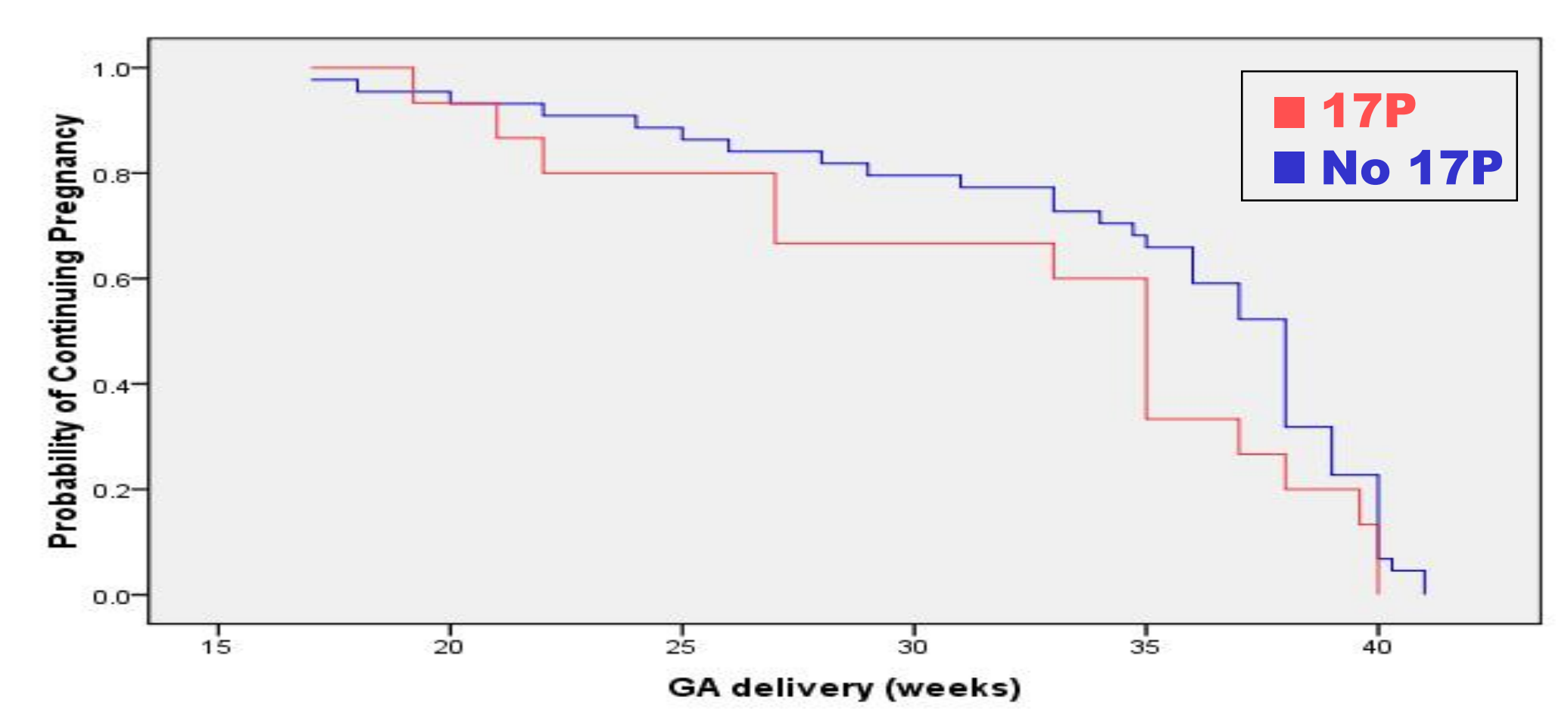

\section{CONCLUSIONS}

Among women with a prior spontaneous PTB and current UIC for CL $<25 \mathrm{~mm}, 17 \mathrm{P}$ did not reduce the rate of PTB $<35$ weeks. -17P did not seem to have a prolonging effect on gestation, nor did it have an effect on neonatal birthweight.

There was a non-significant trend toward increased odds of PTB and lower birthweights in the 17P group compared with the No 17P group. 